Finally, services need to incorporate a strong gender-sensitive and gender-rights perspective, challenging the prevalent 'Marianism' (which glorifies motherhood, while at the same time depicting women as selfless and heroic, willing to put up with the most extreme circumstances in the service of men and children $)^{13}$ with recognition of the different needs of women and of the inequalities they continuously face. ${ }^{14}$ Changes in societal attitudes to motherhood should result in more resources for obstetric care and better recognition of the emotional difficulties women may face in the perinatal period.

\section{Conclusions}

Perinatal mental health has not been significantly researched in South America; violence against women and dire socioeconomic circumstances seem to be implicated by the very high rates of perinatal disorders but research is needed to understand these associations better and to intervene in the most effective way. We need better obstetric services for women, and these should integrate mental healthcare, with especial attention to women and infants at higher risk.

\section{References}

1 Austin, M.-P. (2003) Perinatal mental health: opportunities and challenges for psychiatry. Australasian Psychiatry, 11, 399-403.

2 Lara, M. A., Navarrete, L., Nieto, L., et al (2015) Prevalence and incidence of Perinatal Depression and Depressive Symptoms among Mexican Women. Journal of Affective Disorders, 175, 18-24.

3 Fisher, J., de Mello, M. C., Patel, V., et al (2012) Prevalence and determinants of common perinatal mental disorders in women in low- and lower-middle-income countries: a systematic review. Bulletin of the World Health Organization, 90, 139G-149G.

4 Gelaye, B., Rondon, M. B., Araya, R., et al (2016) Epidemiology of maternal depression, risk factors, and child outcomes in low-income and middle-income countries. Lancet Psychiatry, 3 973-982.

5 Hahn-Holbrook, J., Cornwell-Hinrichs, T. \& Anaya, I. (2018) Economic and health predictors of national postpartum depression prevalence: a systematic review, meta-analysis, and meta-regression of 291 studies from 56 countries. Frontiers Psychiatry, 8, 248 (doi:10.3389/fpsyt.2017.00248).

6 Ortiz Martinez, R. A., Gallego Betancourt, C. X., Buitron Zuñiga, E. L., et al (2016) [Prevalence of positive screen for postpartum depression in a tertiary hospital and associated factors] [Article in Spanish]. Revista Colombiana de Psiquiatría, 45, 253-261.

7 Barrios, Y. V., Gelaye, B., Zhong, Q., et al (2015) Association of childhood physical and sexual abuse with intimate partner violence, poor general health and depressive symptoms among pregnant women. PLoS One, 10(1), e0116609.

8 Ponce de Leon, R. G., Ewerling, F., Serruya, S. J., et al (2019) Contraceptive use in Latin America and the Caribbean with a focus on long-acting reversible contraceptives: prevalence and inequalities in 23 countries. Lancet Global Health, 7(2), e227e235.

9 World Health Organization (2001) Solving mental health problems. In The World Health Report 2001 - Mental Health: New Understanding, New Hope: p. 52. WHO (https://www.who. int/whr/2001/en/whr01_ch3_en.pdf).

10 Saxena, S., Funk, M. \& Chisholm, D. (2013) World Health Assembly adopts Comprehensive Mental Health Action Plan 2013-2020. Lancet, 381, 1970-1971.

11 Rojas, G., Santelices M. P., Martínez P., et al (2015) [Barriers restricting postpartum depression treatment in Chile] [Article in Spanish]. Revista Médica de Chile, 143, 424-432.

12 Kohrt, B. K., Barrueco, S. \& Pérez, C. P. (2015) Domestic violence as a threat to maternal and child well-being in an urban migrant community in Peru. Revista Panamericana de Salud Pública, 37, 265-272.

13 Rondon, M. B. (2003) From Marianism to terrorism: the many faces of violence against women in Latin America. Archives of Women's Mental Health, 6, 157-163.

14 World Health Organization (2014) Health Care for Women Subjected to Intimate Partner Violence or Sexual Violence: A Clinical Handbook: p. 4. WHO.

\section{THEMATIC PAPER}

\title{
Perinatal mental health around the world: priorities for research and service development in the USA
}

\author{
Crystal Edler Schiller, ${ }^{1} \odot$ Matthew J. Cohen ${ }^{2}$ and Michael W. O'Hara ${ }^{3}$
}

${ }^{1}$ Assistant Professor, Department of Psychiatry, University of North Carolina at Chapel Hill, USA

2Postdoctoral Fellow, Department of Psychiatry, University of North Carolina at Chapel Hill, USA

${ }^{3}$ Professor, Department of Psychological and Brain Sciences, University of Iowa, USA. Email: mike-ohara@uiowa.edu

Keywords. Perinatal psychiatry; psychotherapy; anxiety disorders; depressive disorders:

neuroendocrinology.
The perinatal mental health field is growing rapidly, which has yielded innovations in both prevention and treatment. To realise the potential of these innovations to transform clinical practice, further investment in research and clinical service development is required. Clinical services must be expanded by providing increased access to specialty care and education for front-line clinicians. Research is needed to develop a personalised medicine approach to understanding the complex aetiologies of perinatal depression and optimising treatments to promote both remission and long-term recovery. Such initiatives will require policies to prioritise federal research funding and healthcare coverage for perinatal depression.

In the USA, the field of perinatal mental health is experiencing rapid growth to meet the increasing demand for clinical services secondary to 
Policy

- Availability of treatment settings for brexanolone treatment and healthcare coverage

- Psychotherapy for postnatal depression prevention healthcare coverage

- Federal funding for perinatal research

Research

- Innovations in pharmacotherapy and psychotherapy - improving existing treatments with state-of-the-art techniques from other fields

- Identifying the pathophysiology (cellular, neural, genetic and hormonal mechanisms)

- Use of multidimensional symptom assessments for postnatal depression

\section{Clinical services}

- Improved access to specialty care

- Education for front-line clinicians

- Move toward personalised, patient-centred care widespread standardised screening and recognition of mood and anxiety disorders among perinatal women in obstetric and primary care settings. This rapid growth presents unique challenges as well as opportunities to meet the mental healthcare needs of women during what is arguably one of the most vulnerable times in their lives. When and with whom to intervene is a multidimensional challenge facing researchers, clinicians, health systems and policy makers, with implications for both research and service development. Top priorities for policy, research and clinical services for perinatal depression (PND) in the USA will be described in this article, an overview of which is provided in Box 1 .

\section{Current trends in perinatal mental health in the USA}

PND and anxiety are collectively seen as a major public health problem in the USA. The American College of Obstetricians and Gynecologists, the American College of NurseMidwives and the US Preventive Services Task Force recommend universal screening for pregnant and postpartum women during routine obstetric care. Although universal screening is an important step in addressing pervasive mental health concerns among perinatal women, it does not guarantee universal access to treatment. In the USA, the majority of pregnant women with depressive symptoms do not receive mental healthcare. ${ }^{1}$ Prescription medication is the most common form of mental health treatment among pregnant women. ${ }^{1}$ Barriers to care include financial constraints, lack of access to qualified mental health providers, pragmatic issues (e.g. lack of transportation, child care, ability to leave work) and stigma. ${ }^{1,2}$ Researchers, clinicians and policy makers are working to address barriers to accessing effective, high-quality mental health treatment.

\section{Service development Psychotherapy for prevention and treatment of PND}

Existing research literature should guide the expansion of treatment and prevention efforts. From a prevention standpoint, family physicians in the USA are encouraged to engage in preconception planning with women of reproductive age before they start trying to conceive; this intervention is designed as a health education tool intended to promote physical and emotional wellbeing in mothers before pregnancy, to improve the likelihood of positive perinatal outcomes. ${ }^{3}$ Among women who do become pregnant and experience PND symptoms, the majority report that they would prefer to engage in psychotherapy. ${ }^{4}$ Psychotherapy effectively prevents the onset of PND, yet access to affordable, evidence-based psychotherapy is extremely limited in the USA. Widespread adoption of preventive therapy will require routine screening of both current symptoms and past episodes of mental illness, as well as payers to allow mental health providers to bill for preventative services, which would make it possible for existing treatment programmes to expand services to well women. Equipping treatment providers with the skills to implement evidence-based diagnosis, case conceptualisation, treatment and an understanding of the importance of evidencebased care is a critical step toward expanding access to high-quality treatment. Postpartum Support International has developed a curriculum for nurses and therapists to improve access to training for front-line treatment providers. In addition, evidence-based treatments may be delivered online by capitalising on the availability of widespread high-speed internet access, although the efficacy of such programmes have yet to be established. Further, home-visiting programmes for at-risk families during pregnancy and into early childhood are ubiquitous in the USA. The US Health Resources and Services Administration awarded States and organisations almost 
$\$ 500000000$ in the past fiscal year to provide health, social and often mental health services to at-risk families. Programme models include the Nurse-Family Partnership, Parents as Teachers and Healthy Beginnings, among others (https:// mchb.hrsa.gov/maternal-child-health-initiatives/ home-visiting-overview). These interventions are often delivered by non-mental health professionals such as nurses and home visitors. ${ }^{5,6}$ The evidence for the superiority of these interventions has been mixed, which has been partially attributable to the success of usual care provided by home-visiting programmes and the occasional high attrition rate in trials.

\section{Pharmacotherapy for PND}

Another important aspect of clinical care is access to specialised pharmacotherapy for PND. Most women with PND seek treatment from an obstetrician or family physician. However, many of these providers have not received training to weigh the risks and benefits of various medications for pregnant and breastfeeding women, which can be problematic for women experiencing a range of psychopathology during the perinatal period. For example, women with a history of bipolar disorder are ideally monitored during pregnancy by their mental health providers. However, many find themselves in a position where their non-perinatal psychiatrist is reluctant to prescribe mood stabilisers during pregnancy and their obstetrician feels uncomfortable prescribing psychiatric medications other than antidepressants. As such, the expansion of training opportunities for physicians is critical. At University of North Carolina at Chapel Hill, researchers are implementing a consultation warmline for physicians across the state of North Carolina to address questions about diagnosis and medical management of complex cases based on Nancy Byatt's Massachusetts Child Psychiatry Access Program for Moms model. ${ }^{7}$ Further, telehealth has the potential to provide patients who live outside areas with urban, academic centres with access to specialists; the difficulty will be in determining which patients most need specialised care and which patients can be adequately managed by their existing care teams. In the absence of a nationalised healthcare system in the USA, carefully triaging patients based on symptoms and mental health history, increasing access to telehealth services, and training and consultation for community providers may be the best options for increasing access to high-quality perinatal psychiatric care. Efforts by the National Curriculum on Reproductive Psychiatry to institute a standardised curriculum for educating psychiatrists about reproductive-hormone-related mood and anxiety disorders and parallel efforts by Postpartum Support International to train nurse practitioners and therapists are critical for ensuring access to evidence-based care for perinatal women. Finally, the number of intensive out-patient programmes, partial hospitalisation programmes and specialised in-patient units has increased in the past 10 years, but remain limited. Intensive treatment services are costly, which is the main limiting factor preventing widespread adoption of this model of care. Intensive treatment settings are critical, however, for stabilising those in acute crisis (i.e. defined by suicidality, homicidality or psychosis), ensuring the safety of both mothers and babies, and providing a context for the provision of novel, fast-acting therapeutics like brexanolone $^{8}$ or ketamine. ${ }^{9}$ Advocacy for funding these efforts, through hospitals or third-party payers, is needed.

\section{Patient-centred care: case conceptualisation and advocacy}

Increasingly, research suggests that perinatal psychopathology is not confined to depression, but rather spans the full diagnostic nomenclature. By prioritising a woman's perinatal status over her specific diagnoses, however, clinicians risk providing suboptimal treatment. Research is needed to determine the best course of treatment for perinatal women presenting with depression and comorbid obsessive-compulsive disorder and post-traumatic stress disorder symptoms. Perinatal women deserve comprehensive assessment, diagnosis and case conceptualisation to identify primary and secondary treatment targets, just as would occur in any other population. In addition, perinatal women of colour face significant discrimination in obstetric care that results in an increased incidence of negative outcomes, including maternal death. ${ }^{10}$ Women of colour also face significant barriers to mental healthcare. ${ }^{11}$ Perinatal mental health providers must therefore work to subvert stigma and discrimination faced by our patients of colour, both by advocating to ensure optimal obstetric care and by eliminating barriers to culturally competent, unbiased mental healthcare.

\section{Research initiatives \\ Novel therapeutics}

Research on perinatal mental health is flourishing - twice as many academic papers were published on the topic in the past year compared with 2008 - yet perinatal mental health is often siloed from other areas of psychological science, including social, cognitive and behavioural science, that could inform both aetiological models and existing treatment paradigms. For example, the theoretical foundation of interpersonal psychotherapy, one of the most well-validated treatments for PND, is Bowlby's attachment theory. Although the provision of treatment (e.g. setting, number of sessions, format) has changed since its inception, the method and techniques have remained the same. Treatments should not stagnate, however, as research is continually identifying new and better ways to improve attachment and enhance well-being. Therapy outcomes achieved with interpersonal psychotherapy for PND could potentially be improved by using validated techniques that strengthen relationships, ${ }^{12}$ bolster social 
support ${ }^{13}$ and improve mother-infant bonding. ${ }^{14}$ In addition, there is increasing recognition that PND is characterised by significant comorbidity ${ }^{15,16}$ including post-traumatic stress disorder, substance use, obsessions, compulsions, social phobia and personality pathology, that may not be well addressed by standard first-line treatments. As such, research is needed to develop transdiagnostic, flexible interventions that can effectively address both the substantial comorbidity and the range of severity of symptoms that women with PND experience. ${ }^{15}$

Beyond psychotherapy, many questions remain about how best to weigh the risks and benefits of existing pharmacotherapies for perinatal mood and anxiety disorders. Novel digital decision tools may help patients and their physicians effectively and efficiently navigate these difficult decisions. ${ }^{17}$ Moreover, there is increasing recognition among funding agencies that rigorous, highquality patient and infant outcome research is needed, especially for the most commonly prescribed pharmacotherapies. The development of intravenous brexanolone as a novel, fast-acting treatment of postpartum depression was an important scientific breakthrough ${ }^{8}$; however, it is just a starting point. Recent advances in the development of novel therapeutics for postpartum depression, and severe depression more generally, should be leveraged to identify additional treatments that are not only fast-acting but also affordable and safe to administer in patients' homes. The long-term efficacy of brexanolone and its combination with other therapeutics (i.e. pharmacotherapy, psychotherapy or transcranial magnetic stimulation) also must be investigated to identify the best way to sustain long-term recovery.

\section{Identifying pathophysiology and improving symptom detection}

Perhaps more important than identifying new and better treatments for PND is identifying methods by which illness expression can be prevented in the first place. Prevention relies on the ability to identify which women are at highest risk for PND and the nature of the pathophysiology so that early, personalised intervention may be delivered. Despite decades of research aimed at identifying the neuropathophysiology of PND, it remains poorly understood. One reason for our lack of progress is that PND is a heterogeneous diagnosis with multiple aetiologic pathways. The development of personalised interventions will require the identification of specific triggers of affective state change in PND. Existing research suggests that for some women, the hormone changes that accompany childbirth are sufficient to trigger significant affective dysregulation, ${ }^{18}$ and this may represent a specific hormone-sensitive phenotype of PND. ${ }^{19}$ The early identification of hormone sensitivity through stable biomarkers - cellular, neural or genetic - may therefore allow for the development of novel therapeutics designed to prevent affective dysregulation triggered by puerperal hormonal changes.

Another imperative in PND research is to broaden our scope of symptom detection. Many studies conceptualise PND as a unitary construct; however, perinatal affective dysregulation not only includes dysphoria, but also irritability and somatic symptoms, ${ }^{20}$ which are often largely ignored in the research literature. The adoption and use of high-quality, empirically derived measures of mood and anxiety are critical for identifying the pathophysiology and improving opportunities for personalised care.

Finally, research must begin to address the most severe forms of perinatal mood disorders, including severe depression with suicidality, bipolar disorder and psychosis. Severe manifestations of PND are thought to be relatively rare; however, broad-based screening for manic and psychotic symptoms has yet to be implemented in the USA, and so the prevalence is largely unknown. Moreover, postpartum psychosis has been used as an umbrella term that encompasses both severe depression with psychotic features, mania and first-onset psychotic disorders, ${ }^{21}$ which are difficult to differentiate in acutely ill patients. The lack of a specific set of criteria by which to assess and diagnosis postpartum psychosis has hampered systematic research of pathophysiology and potential treatments.

\section{Conclusion}

In summary, it is an exciting time to engage in clinical care and research on PND. Both areas are rapidly expanding, and successful large-scale research efforts have led to widespread adoption of PND screening in obstetric and primary care settings. The next step is to improve access to high-quality mental healthcare by providing training to clinicians across the country, leveraging digital technologies and better understanding the unique needs of perinatal women. Research aimed at understanding the neurobiology of PND has the potential to powerfully enhance personalised approaches to care. Recent successes in developing novel therapeutics and acknowledging the efficacy of targeted prevention services for PND should translate to increased federal funding for perinatal mental health research.

\section{Author contributions}

C.E.S. and M.W.O'H. conceptualised the paper. C.E.S., M.J.C. and M.W.O'H. revised the paper, gave final approval of the draft submitted for publication, and are accountable for all aspects of the work in ensuring that questions related to the accuracy or integrity of any part of the work are appropriately investigated and resolved.

\section{Funding}

This work was supported by the National Institute of Mental Health (grant K23-MH105569 to C.E.S.) and the Foundation of Hope (C.E.S.) 


\section{Declaration of interest \\ None.}

ICMJE forms are in the supplementary material, available online at https://doi.org/10.1192/bji.2020.15.

\section{References}

1 Sanmartin MX, Ali MM, Chen J, Dwyer DS. Mental health treatment and unmet mental health care need among pregnant women with major depressive episode in the United States. Psychiatr Serv 2019; 70(6): 503-6.

2 Keefe RH, Brownstein-Evans C, Polmanteer RSR. Addressing access barriers to services for mothers at risk for perinatal mood disorders: a social work perspective. Soc Work Health Care 2016; 55(1): 1-11

3 Farahi N, Zolotor A. Recommendations for preconception counseling and care. Am Fam Physician 2013; 88(8): 499-506.

4 Goodman JH. Women's attitudes, preferences, and perceived barriers to treatment for perinatal depression. Birth 2009; 36(1): $60-9$

5 Segre LS, Brock RL, O'Hara MW. Depression treatment for impoverished mothers by point-of-care providers: a randomized controlled trial. J Consult Clin Psychol 2015; 83(2): 314-24.

6 Tandon SD, Ward EA, Hamil JL, Jimenez C, Carter M. Perinatal depression prevention through home visitation: a cluster randomized trial of mothers and babies 1-on-1.] Behav Med 2018; 41(5): 641-52

7 Byatt N, Biebel K, Moore Simas TA, Sarvet B, Ravech M, Allison J, et al Improving perinatal depression care: the Massachusetts Child Psychiatry Access Project for Moms. Gen Hosp Psychiatry 2016; 40: 12-7.

8 Meltzer-Brody S, Colquhoun H, Riesenberg R, Epperson CN Deligiannidis KM, Rubinow DR, et al Brexanolone injection in postpartum depression: two multicenter, double-blind, randomized, placebo-controlled, phase 3 trials. Obstet Gynecol Surv 2019; 74(4): 219-20.

9 Murrough JW, Soleimani L, DeWilde KE, Collins KA, Lapidus KA, Iacoviello BM, et al Ketamine for rapid reduction of suicidal ideation: a randomized controlled trial. Psychol Med 2015; 45 (16): $3571-80$

10 Holdt Somer SJ, Sinkey RG, Bryant AS. Epidemiology of racial/ ethnic disparities in severe maternal morbidity and mortality. Semin Perinatol 2017; 41(5): 258-65.
11 Chang J], Tabet M, Elder K, Kiel DW, Flick LH. Racial/ethnic differences in the correlates of mental health services use among pregnant women with depressive symptoms. Matern Child Health J 2016; 20(9): 1911-22.

12 Algoe SB, Kurtz LE, Hilaire NM. Putting the "you" in "thank you": examining other-praising behavior as the active relational ingredient in expressed gratitude. Soc Psychol Personal Sci 2016: 7(7): 658-66.

13 Kok BE, Coffey KA, Cohn MA, Catalino LI, Vacharkulksemsuk T, Algoe SB, et al How positive emotions build physical health: perceived positive social connections account for the upward spiral between positive emotions and vagal tone. Psychol Sci 2013; 24(7): 1123-32.

14 Kochanska G. Mutually responsive orientation between mothers and their young children: implications for early socialization. Child Dev 1997; 68(1): 94-112.

15 Grote NK, Katon WJ, Russo JE, Lohr MJ, Curran M, Galvin E, et al A randomized trial of collaborative care for perinatal depression in socioeconomically disadvantaged women: the impact of comorbid posttraumatic stress disorder. J Clin Psychiatry 2016; 77(11): 1527-37.

16 Falah-Hassani K, Shiri R, Dennis C-L. Prevalence and risk factors for comorbid postpartum depressive symptomatology and anxiety. J Affect Disord 2016; 198: 142-7.

17 Vigod S, Hussain-Shamsy N, Grigoriadis S, Howard LM, Metcalfe K, Oberlander TF, et al A patient decision aid for antidepressant use in pregnancy: study protocol for a randomized controlled trial. Trials 2016; 17(1): 110.

18 Bloch M, Schmidt PJ, Danaceau M, Murphy J, Nieman L, Rubinow DR. Effects of gonadal steroids in women with a history of postpartum depression. Am J Psychiatry 2000; 157(6): 92430

19 Schiller CE, Meltzer-Brody S, Rubinow DR. The role of reproductive hormones in postpartum depression. CNS Spectr 2015; 20(1): 48-59.

20 Williamson JA, O'Hara MW, Stuart S, Hart KJ, Watson D. Assessment of postpartum depressive symptoms: the importance of somatic symptoms and irritability. Assessment 2015; 22(3): 309-18.

21 Bergink V, Rasgon N, Wisner KL. Postpartum psychosis: madness, mania, and melancholia in motherhood. Am J Psychiatry 2016; 173(12): 1179-88.

\title{
SPECIAL PAPER \\ Scaling up mental health services in Sudan: Sudanese psychiatrists' opinions
}

\author{
Abdelgadir Hussein M. Osman, $\odot$ Aisha Bakhiet, Samia Elmusharaf, \\ Abdelaziz Omer and Abdalla Abdelrahman
}

Department of Psychiatry, Faculty of Medicine, University of Khartoum, Khartoum, Sudan Email: abdelgadir1159@yahoo. com

Keywords. Scaling up mental health services; capacity building; mental health; Sudan.

First received 11 Oct 2019 Final revision 6 Mar 2020 Accepted 9 Mar 2020
We invited 108 psychiatrists of Sudanese origin, working in and outside Sudan, to take part in a study looking at the most appropriate method for scaling up mental health services in Sudan. Of those psychiatrists who were approached, 81 (75\%) responded. Among the respondents, $30(37 \%)$ resided and worked in Sudan, and 51 (63\%) worked outside Sudan (mostly in the UK and Arab Gulf States). Most respondents preferred the lay counsellor model $(43,53.2 \%)$ to address the current shortage of human resources for scaling up mental health services.

\section{Background}

Several World Health Organization (WHO) reports have issued calls for low- and 